\title{
Hotspots of sensitivity to GCM biases in global modelling of mean and extreme runoff.
}

L.V. Papadimitriou ${ }^{1}$, A.G. Koutroulis ${ }^{1}$, M.G. Grillakis ${ }^{1}$, I.K. Tsanis ${ }^{1,2}$

${ }^{1}$ Technical University of Crete, School of Environmental Engineering, Chania, Greece

${ }^{2}$ McMaster University, Department of Civil Engineering, Hamilton, ON, Canada

Correspondence to: I. K. Tsanis (tsanis@hydromech.gr)

\section{Supplement}
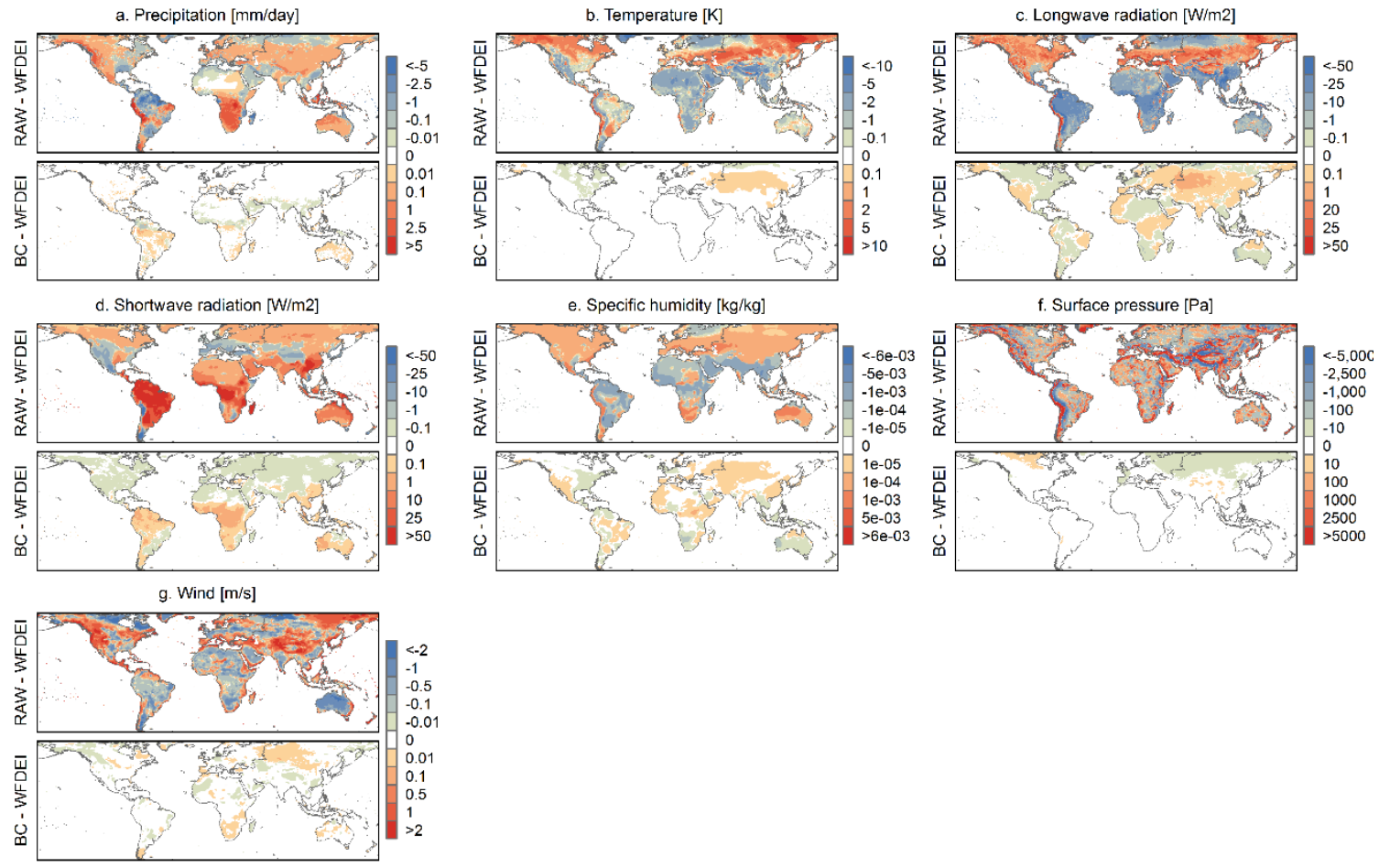

Figure S 1. 1981-2010 December, January, February Averages (DJF) of Forcing Data, differences of WFDEI from raw and bias adjusted input. 

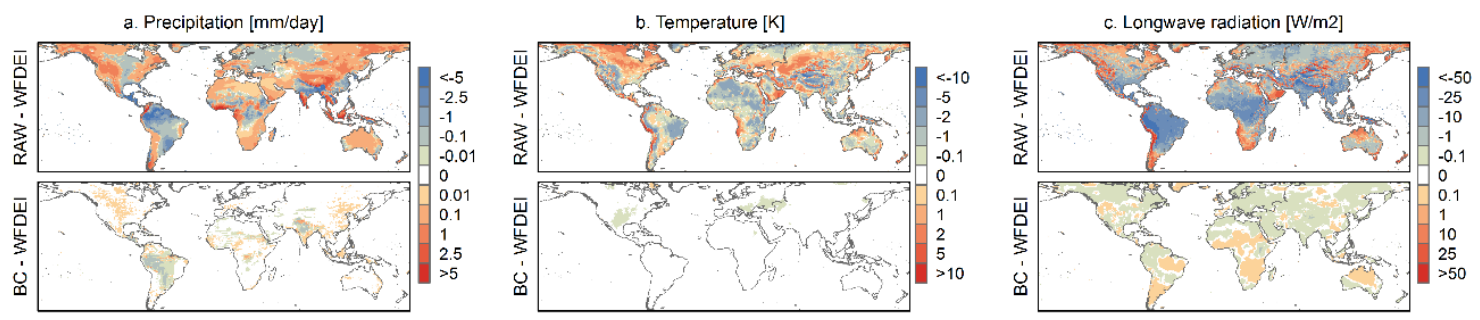

d. Shortwave radiation $[\mathrm{W} / \mathrm{m} 2]$
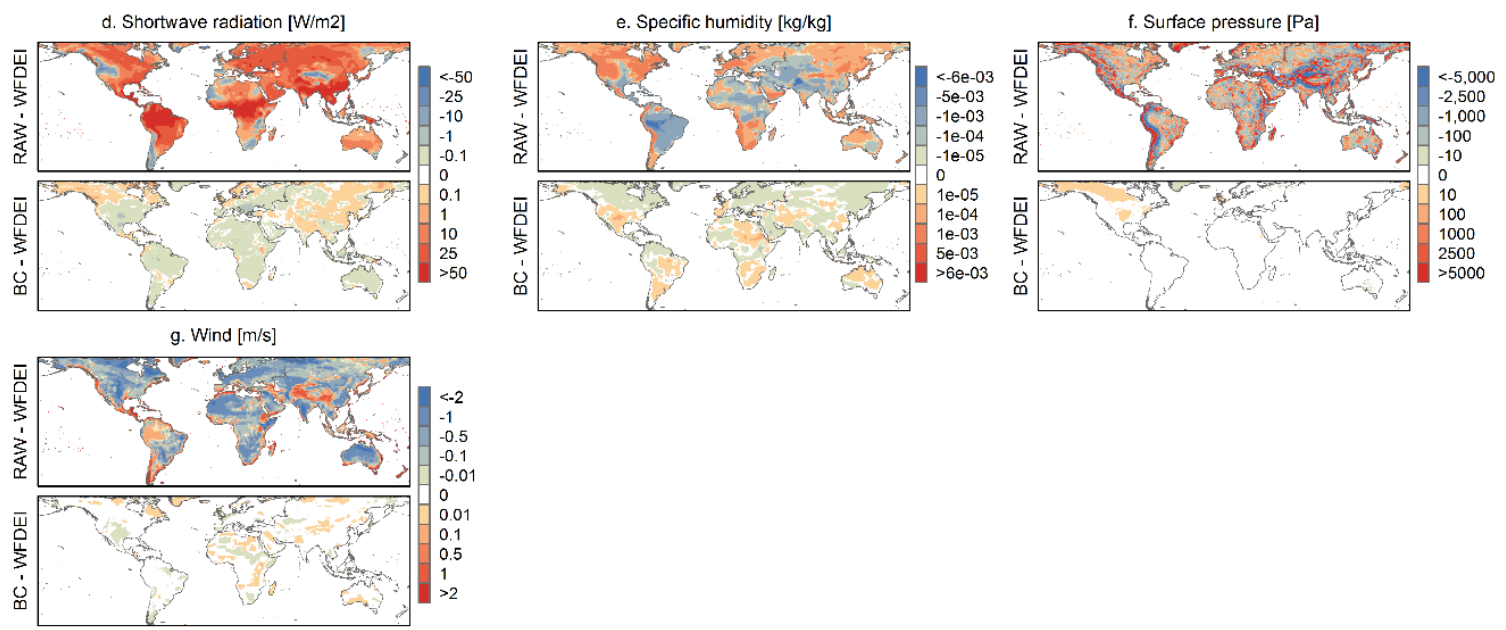

Figure S 2. 1981-2010 June, July, August Averages (JJA) of Forcing Data, differences of WFDEI from raw and bias adjusted input.

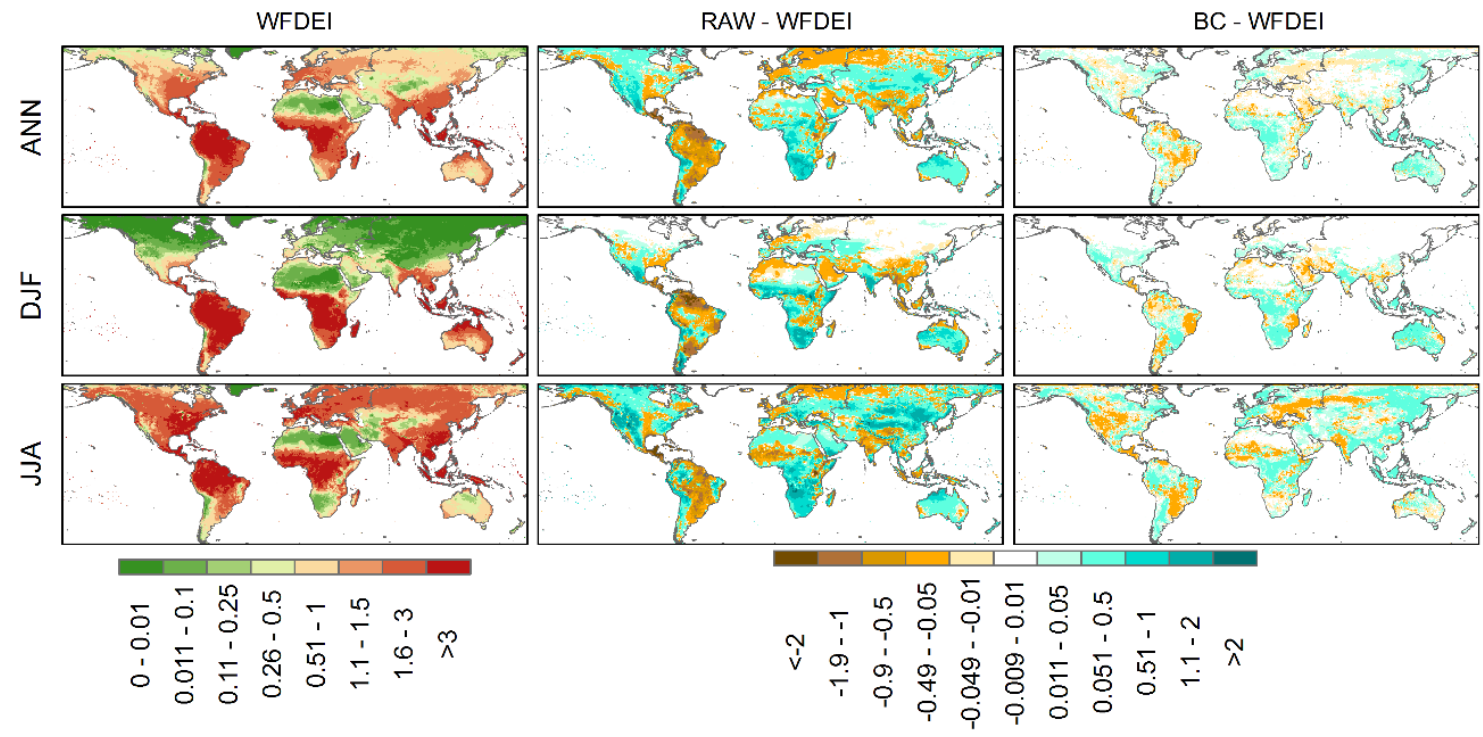

Figure S 3. Evapotranspiration [mm/day], for WFDEI input forcing (left column), and differences in evapotranspiration forced the GCM ensemble and WFDEI, for raw (middle column) and bias adjusted (left column) forcing. Results are shown for ANN, DJF and JJA averages of the 1981-2010 period. 

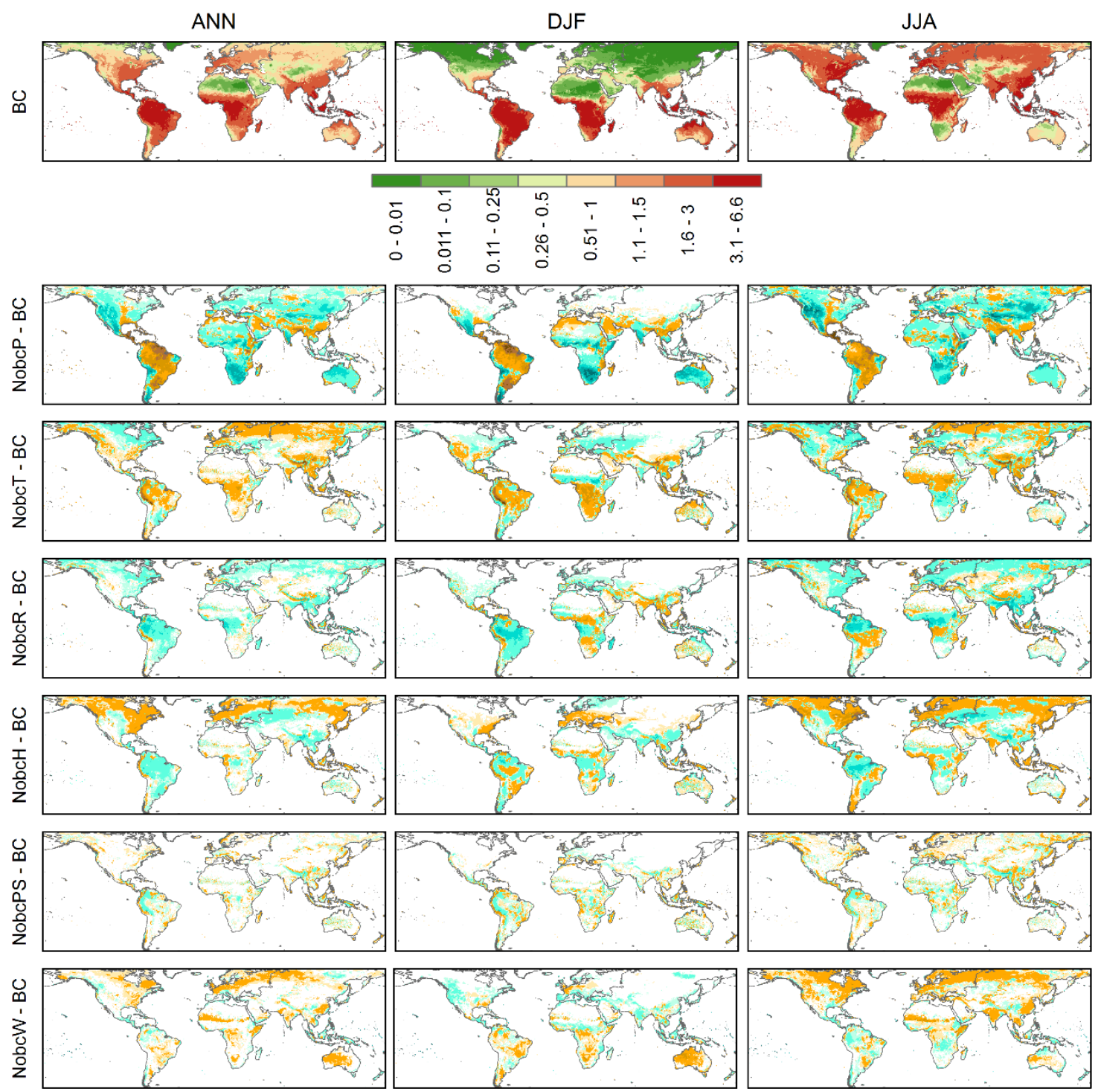

$$
\begin{aligned}
& \text { - } \\
& \text { V่ }
\end{aligned}
$$

Figure S 4. Evapotranspiration [mm/day], from all bias corrected GCM ensemble forcing (top row), and differences in evapotranspiration forced with one variable uncorrected and all bias corrected ensemble. Results are shown for ANN, DJF and JJA averages of the 1981-2010 period. 

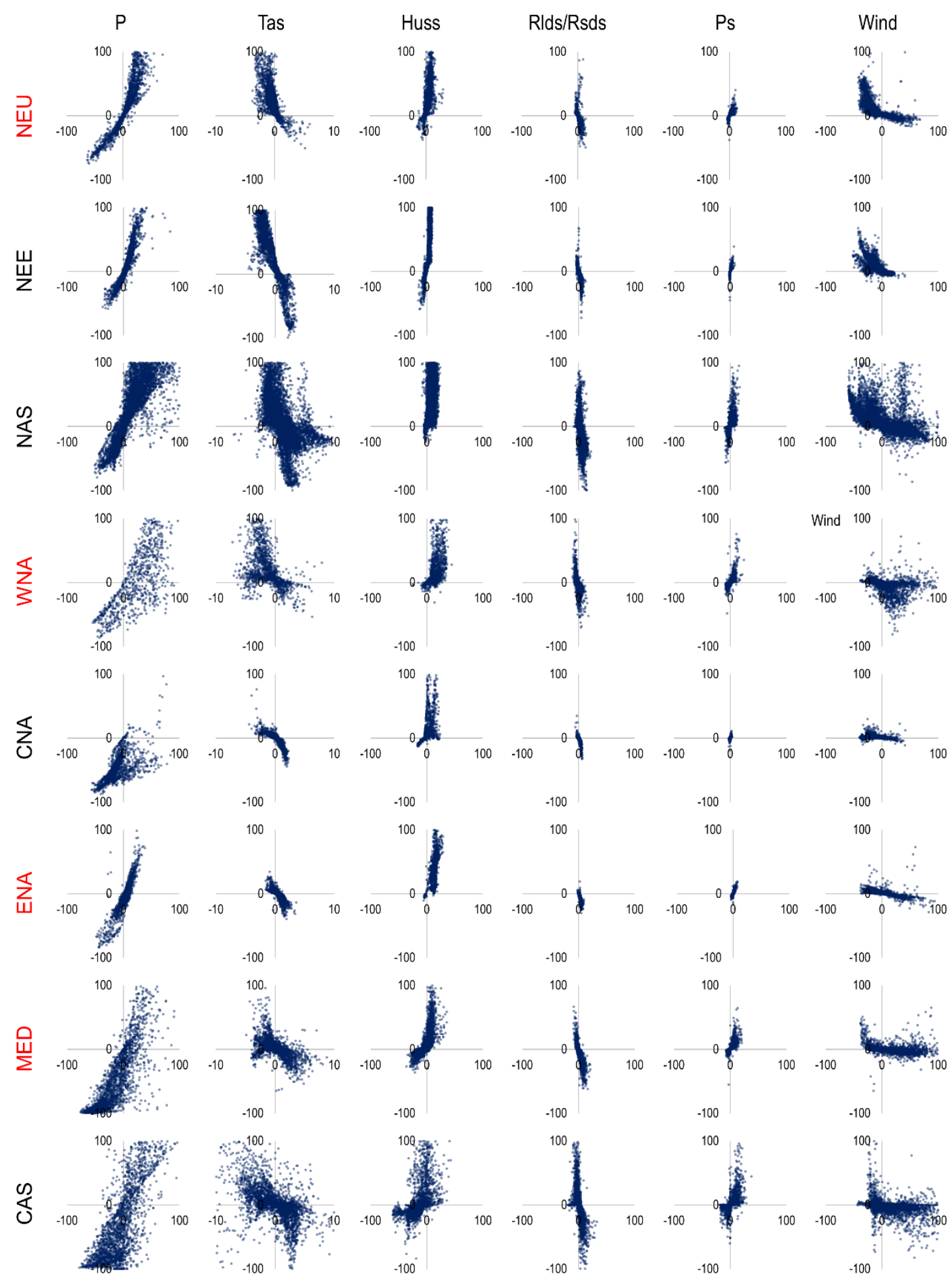

Figure S 5. Scatterplots of percent bias of a non-bias corrected variable and its effect on output runoff. Horizontal axis expresses the percent bias of the input variable and vertical axis expresses the change in output runoff caused by the input variable bias. Scatterplots are shown for the input variables P, Tas, Huss, Rlds/Rsds, Ps and Huss and for 24 land regions. 

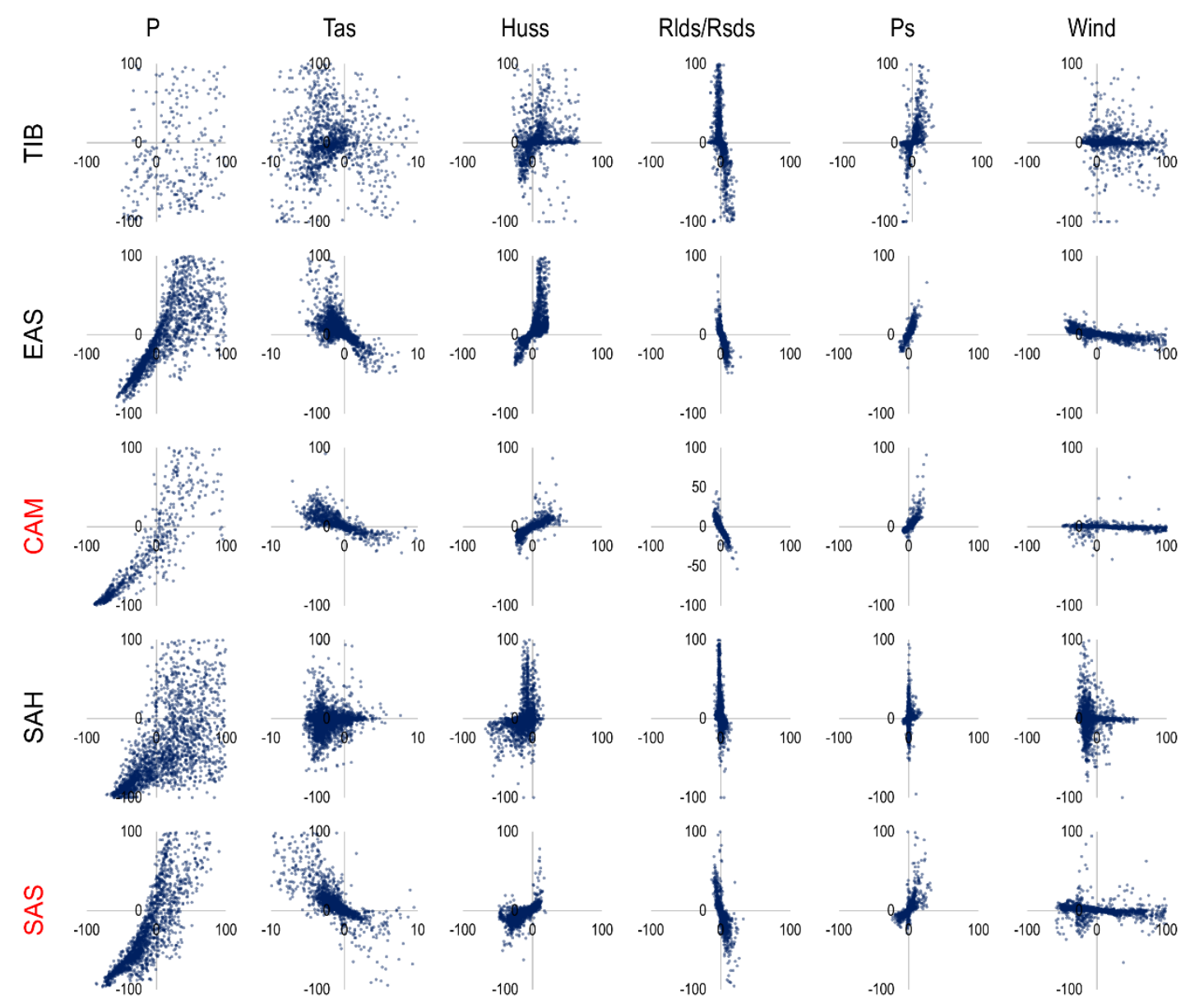

$$
-100 \div . .
$$
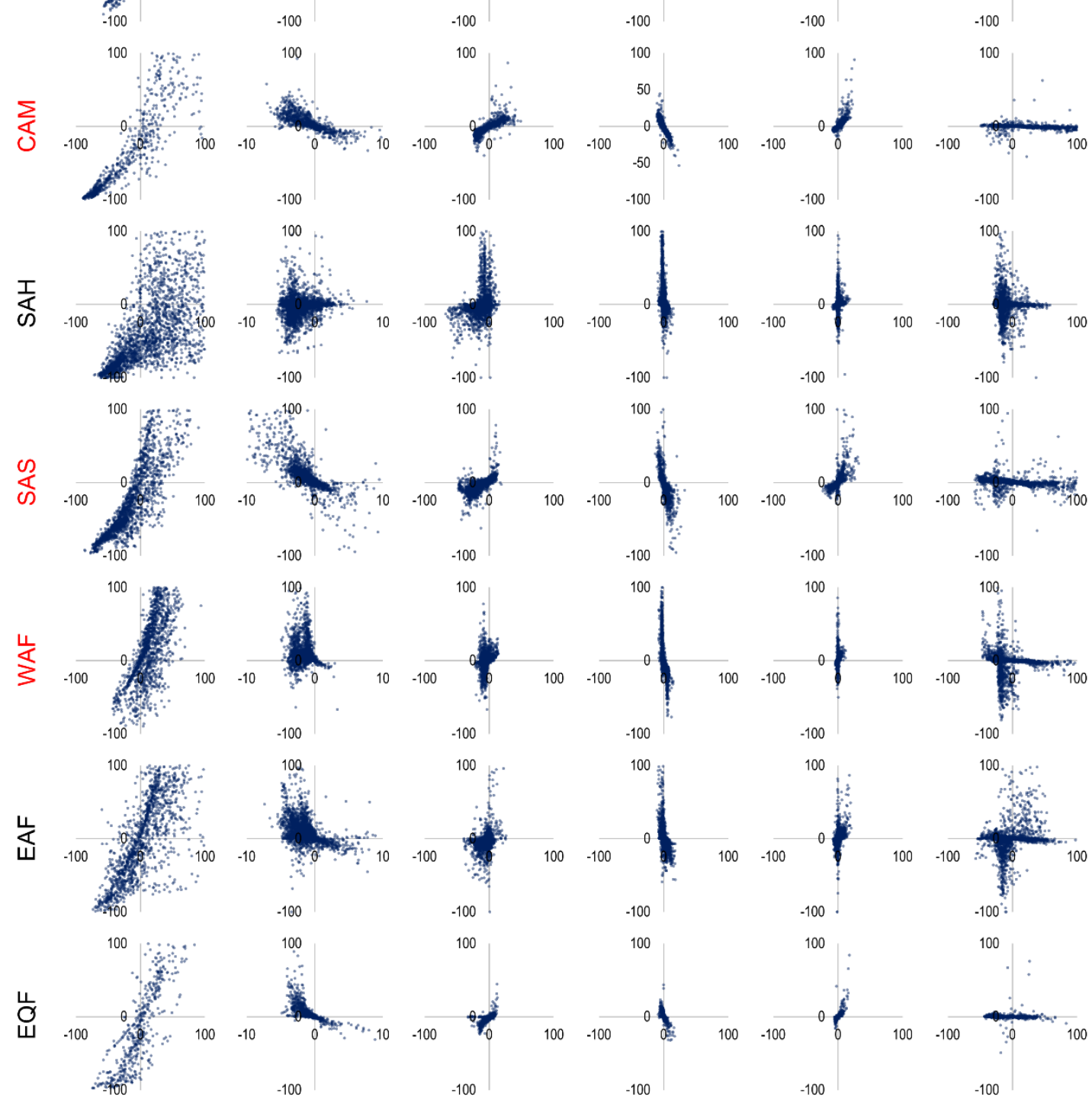

Figure S 5 (continued). 

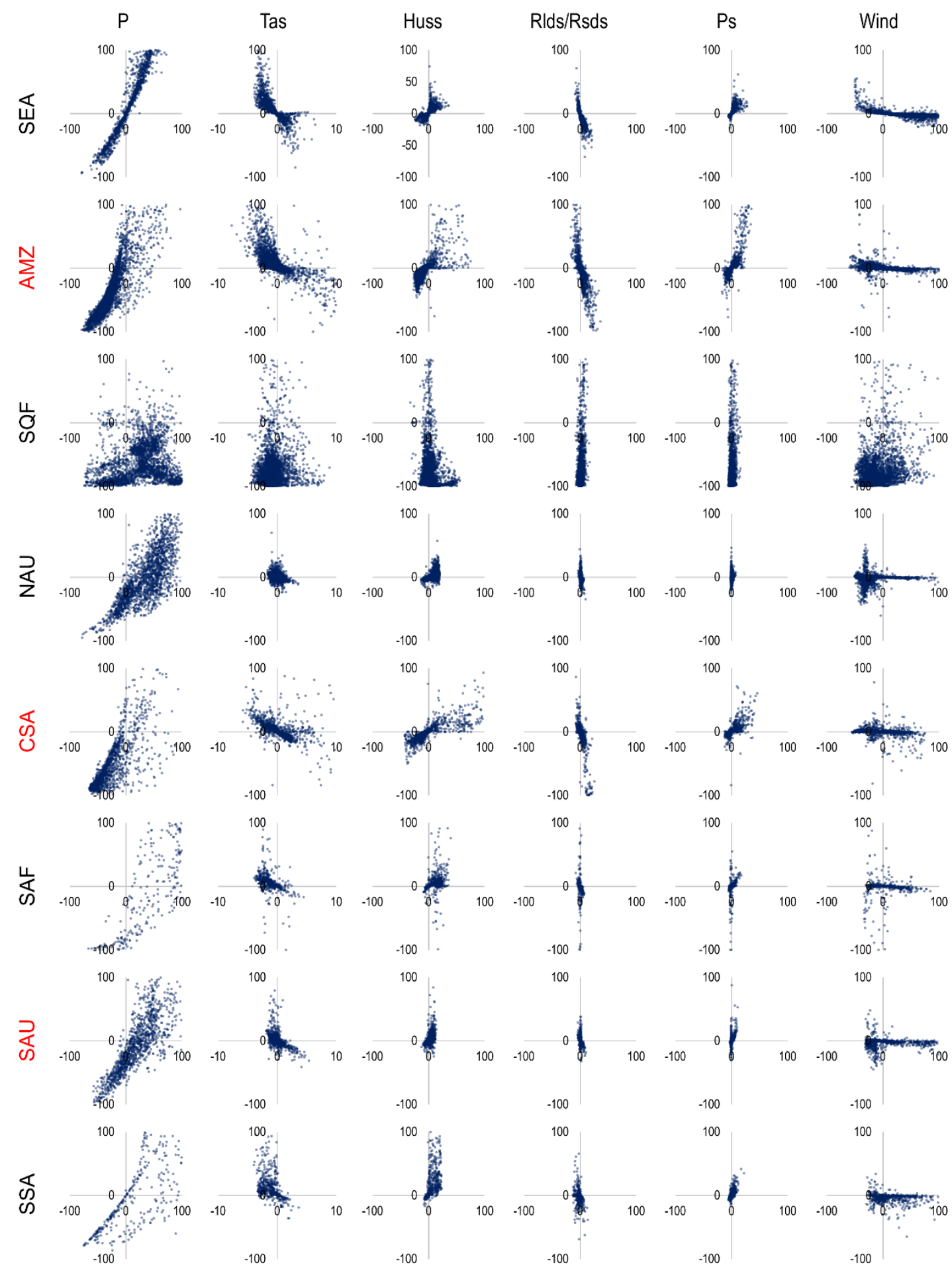

Figure S 5 (continued). 

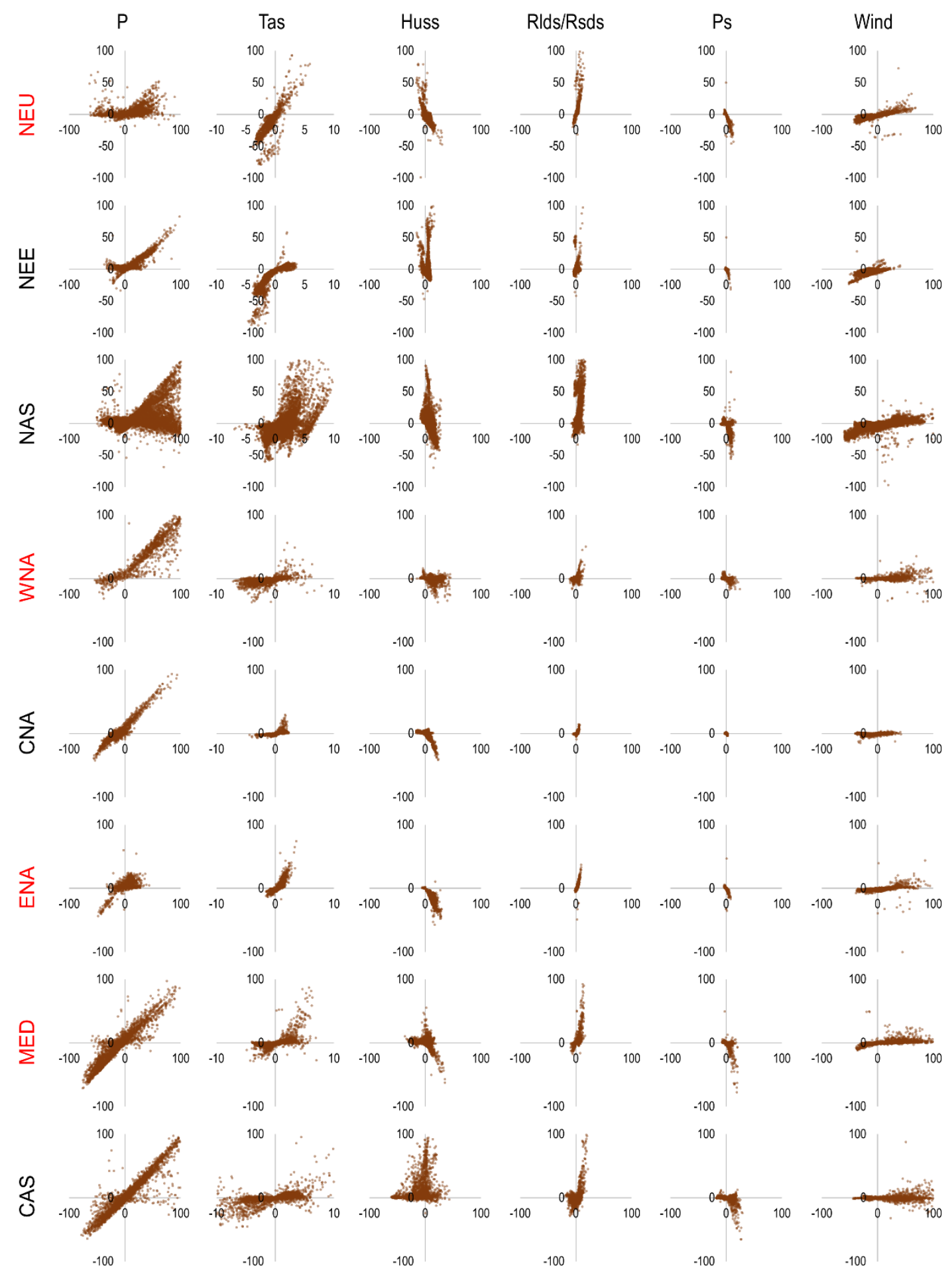

Figure S 6. Scatterplots of percent bias of a non-bias corrected variable and its effect on output ET. Horizontal axis expresses the percent bias of the input variable and vertical axis expresses the change in output runoff caused by the input variable bias. Scatterplots are shown for the input variables P, Tas, Huss, Rlds/Rsds, Ps and Huss and for 24 land regions. 

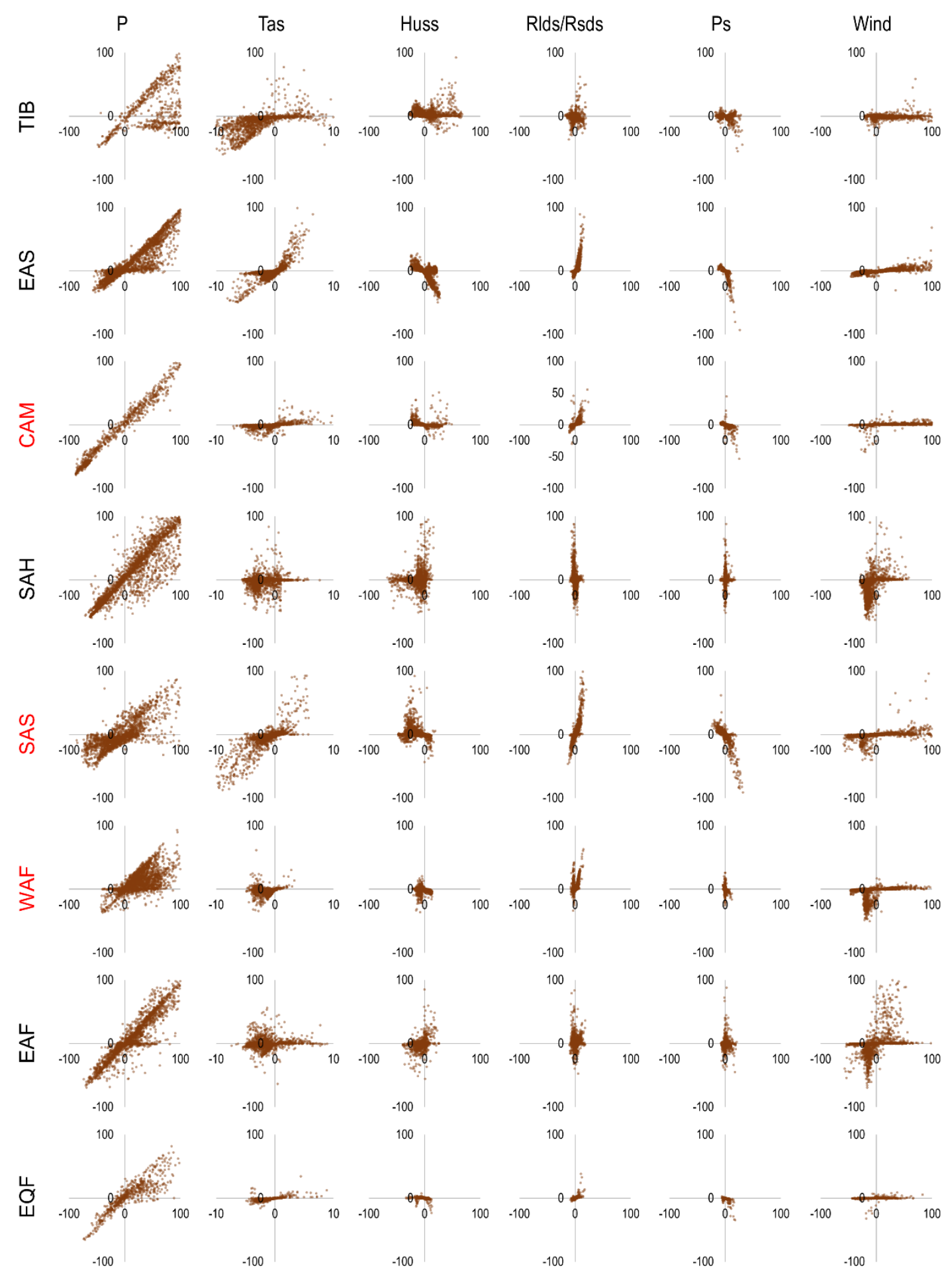

Figure S 6 (continued). 

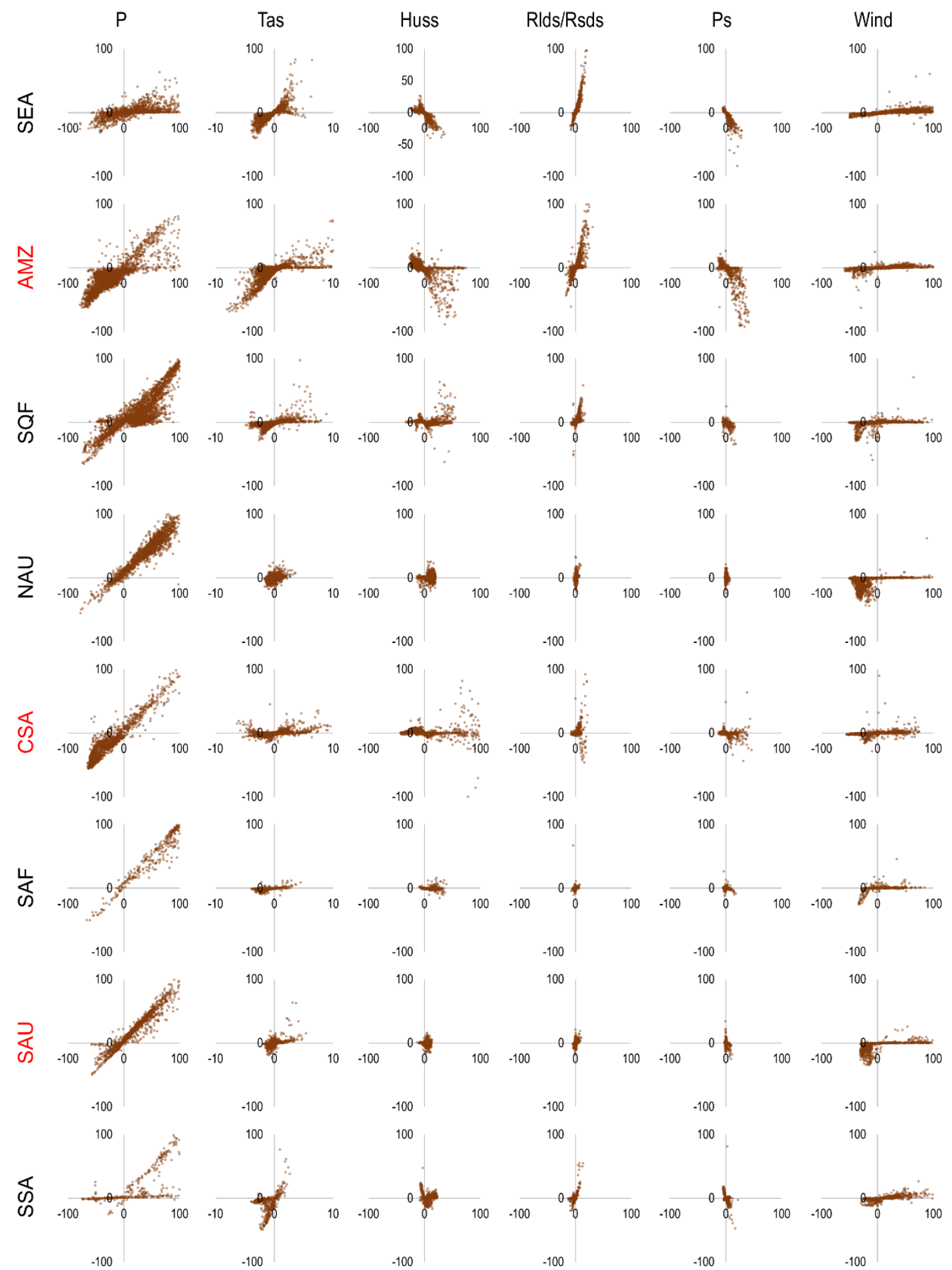

Figure S 6 (continued). 
Table S 1. Percent bias of input variable and resulting change in output evapotranspiration. The median value of all gridboxes of 24 land regions are shown.

\begin{tabular}{|c|c|c|c|c|c|c|c|}
\hline & \% Difference & $\operatorname{Pr}[\mathrm{kg} / \mathrm{m} 2 / \mathrm{s}]$ & Tas [K] & Huss [kg/kg] & $\begin{array}{r}\text { Rlds/Rsd: } \\
{[\mathrm{W} / \mathrm{m} 2]}\end{array}$ & Ps [Pa] & Wind $[\mathrm{m} / \mathrm{s}]$ \\
\hline \multirow[t]{2}{*}{ GLOBAI } & Variable & 14.46 & -0.57 & 0.91 & 1.73 & -0.02 & -5.86 \\
\hline & ET & 4.12 & -1.52 & 0.08 & 0.61 & -0.01 & -0.20 \\
\hline \multirow[t]{2}{*}{ NEU } & Variable & 14.60 & -0.46 & 4.10 & 1.86 & -0.05 & -9.79 \\
\hline & ET & 2.96 & -6.54 & -7.09 & 1.85 & -0.11 & -3.29 \\
\hline \multirow[t]{2}{*}{ NEE } & Variable & 4.89 & -1.44 & 3.32 & 2.44 & 0.10 & -11.77 \\
\hline & $\mathrm{ET}$ & 2.80 & -11.88 & -1.85 & 1.63 & -0.17 & -3.20 \\
\hline \multirow[t]{2}{*}{ NAS } & Variable & 26.05 & 0.67 & 8.05 & 3.53 & -0.06 & -1.08 \\
\hline & $\mathrm{ET}$ & 3.95 & -6.68 & -4.59 & 3.39 & -0.08 & -1.12 \\
\hline \multirow[t]{2}{*}{ WNA } & Variable & 65.92 & -1.75 & 13.55 & -1.23 & 0.14 & 10.23 \\
\hline & $\mathrm{ET}$ & 58.43 & -2.64 & 0.29 & -0.35 & -0.02 & 0.00 \\
\hline \multirow[t]{2}{*}{ CNA } & Variable & -12.84 & 0.11 & 2.29 & 1.68 & -0.08 & -14.79 \\
\hline & ET & -0.43 & -0.52 & 0.47 & 0.59 & -0.01 & -0.49 \\
\hline \multirow[t]{2}{*}{ ENA } & Variable & 4.08 & 0.49 & 13.40 & 2.71 & 0.10 & 5.47 \\
\hline & ET & 2.95 & 0.60 & -16.94 & 2.31 & -0.08 & -1.63 \\
\hline \multirow[t]{2}{*}{ MED } & Variable & -14.39 & -0.15 & -1.34 & 0.55 & 0.41 & 14.94 \\
\hline & ET & -1.40 & -0.15 & 0.20 & 0.18 & -0.05 & 0.29 \\
\hline \multirow[t]{2}{*}{ CAS } & Variable & 6.44 & -0.03 & -13.00 & 1.37 & -0.41 & 8.09 \\
\hline & ET & 3.99 & 0.26 & 1.91 & -0.34 & 0.01 & -0.11 \\
\hline \multirow[t]{2}{*}{ TIB } & Variable & 128.47 & -2.94 & 7.69 & -1.14 & -0.12 & 12.59 \\
\hline & ET & 61.67 & -4.64 & 1.34 & -0.37 & -0.03 & 0.00 \\
\hline \multirow[t]{2}{*}{ EAS } & Variable & 19.25 & -0.94 & 2.92 & 2.51 & -0.20 & -3.55 \\
\hline & ET & 8.36 & -2.20 & -0.18 & 0.82 & 0.00 & -0.14 \\
\hline \multirow[t]{2}{*}{ CAM } & Variable & 11.43 & -0.98 & -6.16 & -0.40 & 0.15 & 25.27 \\
\hline & $\mathrm{ET}$ & 13.70 & -0.60 & 0.66 & 0.12 & -0.01 & 0.24 \\
\hline \multirow[t]{2}{*}{$\mathrm{SAH}$} & Variable & 54.11 & -2.73 & -8.96 & -0.47 & 0.22 & -13.59 \\
\hline & ET & 49.75 & 0.05 & 0.01 & 0.03 & 0.00 & -0.17 \\
\hline \multirow[t]{2}{*}{ SAS } & Variable & -9.19 & -1.08 & -13.11 & 1.39 & -0.05 & -6.81 \\
\hline & ET & -4.88 & -2.64 & 0.61 & 1.94 & 0.00 & -0.40 \\
\hline \multirow[t]{2}{*}{ WAF } & Variable & 26.74 & -1.51 & -5.79 & -0.88 & -0.10 & -15.13 \\
\hline & ET & 9.68 & -1.14 & 0.04 & 0.61 & 0.02 & -0.15 \\
\hline \multirow[t]{2}{*}{ EAF } & Variable & 23.22 & -1.68 & -5.76 & -0.06 & -0.25 & -12.11 \\
\hline & ET & 12.98 & -0.56 & 0.14 & 0.31 & 0.01 & -0.07 \\
\hline \multirow[t]{2}{*}{ EQF } & Variable & 5.64 & -1.55 & -2.15 & -0.25 & -0.20 & -10.09 \\
\hline & ET & 5.53 & -1.17 & 0.22 & -0.19 & -0.04 & 0.00 \\
\hline \multirow[t]{2}{*}{ SEA } & Variable & 19.76 & -0.87 & 0.89 & 1.11 & 0.23 & 34.57 \\
\hline & ET & 0.88 & -4.61 & -1.18 & 2.13 & -0.22 & 1.00 \\
\hline \multirow[t]{2}{*}{$\mathrm{AMZ}$} & Variable & -26.58 & -0.35 & -13.19 & 4.06 & -0.19 & -4.00 \\
\hline & ET & -19.01 & -2.26 & 2.98 & 5.07 & 0.10 & -0.02 \\
\hline \multirow[t]{2}{*}{ SQF } & Variable & 36.45 & -0.90 & 0.89 & 0.90 & -0.03 & -15.60 \\
\hline & ET & 14.83 & -1.16 & 0.04 & 0.11 & 0.00 & -0.09 \\
\hline \multirow[t]{2}{*}{ NAU } & Variable & 41.15 & -0.04 & 7.71 & 1.43 & 0.10 & -28.46 \\
\hline & ET & 41.15 & -0.38 & 0.12 & 0.40 & -0.02 & -1.36 \\
\hline
\end{tabular}




\begin{tabular}{|c|c|c|c|c|c|c|c|}
\hline \multirow[t]{2}{*}{ CSA } & Variable & -32.80 & 0.70 & -11.53 & 3.05 & -0.23 & -7.50 \\
\hline & $\mathrm{ET}$ & -16.97 & 0.75 & 1.55 & 1.23 & 0.03 & -0.23 \\
\hline \multirow[t]{2}{*}{ SAF } & Variable & 89.80 & -1.41 & 14.28 & -0.38 & 0.68 & -4.74 \\
\hline & $\mathrm{ET}$ & 82.76 & -0.57 & -0.29 & -0.08 & -0.03 & 0.06 \\
\hline \multirow[t]{2}{*}{ SAU } & Variable & 18.92 & -0.28 & 2.00 & 0.85 & -0.13 & -11 \\
\hline & ET & 19.88 & -0.10 & -0.07 & 0.06 & -0.01 & -0.12 \\
\hline \multirow[t]{2}{*}{ SSA } & Variable & 72.07 & -1.22 & 5.07 & -1.77 & 0.08 & 9.91 \\
\hline & ET & 25.78 & -3.62 & 1.75 & -0.12 & -0.03 & 0.07 \\
\hline
\end{tabular}

